\title{
Radiofrequency Catheter Ablation for Atrial Fibrillation Elicited "Jackhammer Esophagus": A New Complication Due to Vagal Nerve Stimulation?
}

\author{
Salvatore Tolone, ${ }^{1 *}$ Edoardo Savarino, ${ }^{2}$ and Ludovico Docimo ${ }^{1}$ \\ ${ }^{1}$ Division of Surgery, Department of Surgery, Second University of Naples, Naples, Italy; and ${ }^{2}$ Division of Gastroenterology, Department of \\ Surgery, Oncology and Gastroenterology, University of Padua, Padua, Italy
}

\begin{abstract}
Radiofrequency catheter ablation (RFCA) is a potentially curative method for treatment of highly symptomatic and drug-refractory atrial fibrillation (AF). However, this technique can provoke esophageal and nerve lesion, due to thermal injury. To our knowledge, there have been no reported cases of a newly described motor disorder, the Jackhammer esophagus (JE) after RFCA, independently of GERD. We report a case of JE diagnosed by high-resolution manometry (HRM), in whom esophageal symptoms developed 2 weeks after RFCA, in absence of objective evidence of GERD. A 65-year-old male with highly symptomatic, drug-refractory paroxysmal AF was candidate to complete electrical pulmonary vein isolation with RFCA. Prior the procedure, the patient underwent HRM and impedance-pH to rule out GERD or hiatal hernia presence. All HRM parameters, according to Chicago classification, were within normal limits. No significant gastroesophageal reflux was documented at impedance $\mathrm{pH}$ monitoring. Patient underwent RFCA with electrical disconnection of pulmonary vein. After two weeks, patient started to complain of dysphagia for solids, with acute chest-pain. The patient repeated HRM and impedance-pH monitoring 8 weeks after RFCA. HRM showed in all liquid swallows the typical spastic hypercontractile contractions consistent with the diagnosis of $\mathrm{JE}$, whereas impedance-pH monitoring resulted again negative for GERD. Esophageal dysmotility can represent a possible complication of RFCA for AF, probably due to a vagal nerve injury, and dysphagia appearance after this procedure must be timely investigated by HRM.
\end{abstract}

(J Neurogastroenterol Motil 2015;21:612-615)

Key Words

Atrial fibrillation; Catheter ablation; Esophageal dysmotility; Manometry; Jackhammer esophagus

Received: February 27, 2015 Revised: March 30, 2015 Accepted: April 14, 2015

(c) This is an Open Access article distributed under the terms of the Creative Commons Attribution Non-Commercial License (http://creativecommons. org/licenses/by-nc/4.0) which permits unrestricted non-commercial use, distribution, and reproduction in any medium, provided the original work is properly cited.

${ }^{*}$ Correspondence: Salvatore Tolone, MD, PhD

Division of Surgery, Department of Surgery, Second University of Naples, Via Pansini 5, ZIP 80131, Naples, Italy

Tel: +39-08-15666658, Fax: +39-08-15666658, E-mail: salvatore.tolone@unina2.it

Financial support: None.

Conflicts of interest: None.

Author contributions: Salvatore Tolone contributed to conception and design of the study, and drafting the manuscript; Edoardo Savarino contributed to conception and design of the study, and drafting the manuscript; and Ludovico Docimo gave approval of the final version of the manuscript.

ORCID: Salvatore Tolone, http://orcid.org/0000-0002-1653-9903. 


\section{Introduction}

Radiofrequency catheter ablation (RFCA) is a potentially curative method for treatment of highly symptomatic and drug-refractory atrial fibrillation (AF). However, this technique can provoke esophageal and nerve lesion, due to thermal injury. In particular, esophageal ulcerations and more rarely but potentially life-threatening complication as development of atrio-esophageal fistulas have been reported. ${ }^{1}$ Also, few studies reported on a possible RFCA damage to the vagal plexus surrounding the distal esophagus during left atrial ablation. This damage can result in increased gastroesophageal reflux disease (GERD) and esophageal dysmotility incidence after RFCA. ${ }^{2}$

High-resolution manometry (HRM) is characterized by a higher number of pressure recording sites and a lower distance between them compared with traditional manometry. This technique has been recently shown to objectify and standardize the diagnosis of esophageal motility disorders. In particular, Jackhammer esophagus (JE) is a newly described entity identified and defined as a distal contractile integral (DCI) of greater than $8000 \mathrm{mmHg} \cdot \mathrm{cm} \cdot \mathrm{sec}$ on single or multiple swallows in the presence of normal lower esophageal sphincter (LES) relaxation; this DCI value was not encountered in control subjects. ${ }^{3}$ Also, JE is characterized by repetitive and high-amplitude contractions and it is usually accompanied by dysphagia or chest pain, occurring either in association with esophageal pathology or as an isolated motility disturbance. ${ }^{4}$ Its physiopathology remains unclear; in fact some JE patients showed an associated mechanical outflow obstruction of the esophagogastric junction, others an underlying reflux disease, and finally some are thought to be caused by a primary esophageal muscle hypercontractility.

We report a case of JE diagnosed by HRM, in whom esophageal symptoms developed 2 weeks after RFCA, in absence of objective evidence of GERD at upper endoscopy and reflux monitoring. The etiopathogenesis of this esophageal dysmotility in relation to RFCA will be discussed and reviewed.

\section{Case Report}

Sixty-five-year-old male with highly symptomatic, drug-refractory paroxysmal AF was candidate to complete electrical pulmonary vein isolation (PVI) with RFCA. One month before the procedure, in 2013, cardiologist referred the patient to our Esophageal Motility Lab, at Second University of Naples, to rule out GERD or hiatal hernia presence, as a possible trigger for AF. At visit, he denied any significant past history of heartburn, regurgitation, chest pain or dysphagia, and the filled GerdQ questionnaire scored a value of 0 ; no use of proton pump inhibitors (PPIs) or antiacid drugs was recorded. He underwent HRM and 24-hour esophageal impedance-pH monitoring. HRM study was performed with a $4.2 \mathrm{~mm}$ outer diameter solid state assembly with a 32-channel probe (Sandhill-HRiM catheter InSight; Sandhill Scientific Inc, Highlands Ranch, CO, USA). Data acquisition, display, and analysis were performed using dedicated software (Sandhill Bioview; Sandhill Scientific Inc), after a proper thermal compensation. All HRM parameters, according to Chicago classification for esophageal motility, were within normal limits (Fig. 1). Ambulatory impedance $\mathrm{pH}$ monitoring study was performed after an overnight fast, on an out-patient basis, using a ZepHr system (Sandhill Scientific Inc). A dedicated catheter (with intraluminal impedance segments positioned at 3, $5,7,9,15$, and $17 \mathrm{~cm}$ above the LES) was placed transnasally, with the esophageal $\mathrm{pH}$ sensor positioned $5 \mathrm{~cm}$ above the manometrical determined LES. No symptom was reported and no significant gastro-esophageal reflux was documented in pre-RFCA 24-hour esophageal impedance-pH monitoring.

Patient underwent PVI in January 2014. Mapping and ablation were performed using a $3.5 \mathrm{~mm}$ open irrigated-tip quadripolar catheter (Therapy Cool Path, Irvine Biomedical, Irvine, CA, USA), with a maximum energy delivery of $25 \mathrm{~W}$ at posterior

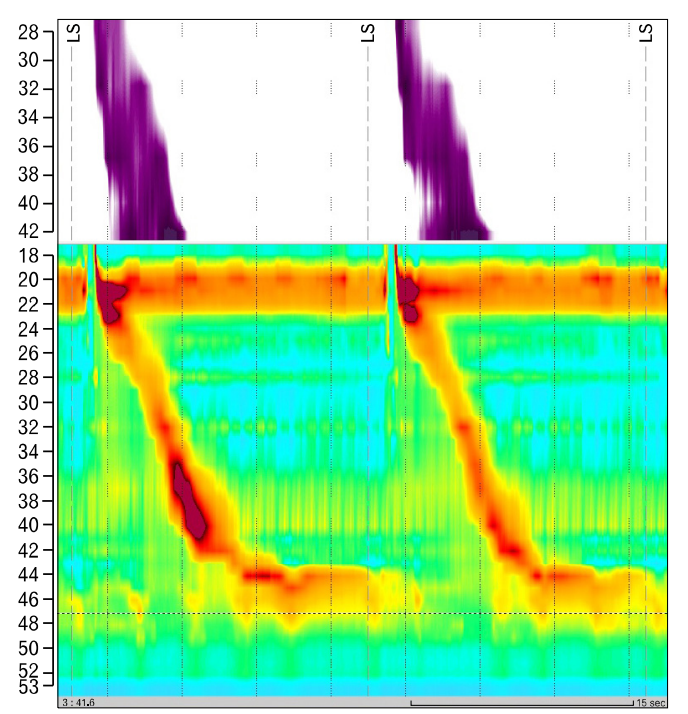

Figure 1. High-resolution manometry trace before radiofrequency catheter ablation for atrial fibrillation. Esophageal peristalsis was normal, according to Chicago classification. 
left atrial wall. Electrical disconnection of pulmonary vein from left atrial was documented. Hospital course was uneventful; INR value of 2-3 was reached and maintained for at least 3 months; PPI $20 \mathrm{mg}$ twice daily were prophylactically administered to avoid gastric injuries. Anti-arythmic drug therapy was stopped 4 weeks after ablation. After 2 months, patient started to complain of dysphagia for solids, with acute chest-pain, without any heartburn or regurgitation. Finally, he underwent cardiological follow-up. Conventional electrocardiography, 48-hour Holter-electrocardiography, serological analysis, transthoracic echocardiography, chest $\mathrm{X}$-ray and cardiac magnetic resonance imaging were normal. Due to concomitant weight-loss (5 $\mathrm{kg}$ in one month), patient repeated the upper endoscopy carried out by the same operator of the previous one (ST). Esophageal mucosa was normal, no tear or erosion was recorded. However, an hypercontractility of esophageal wall was noted. Based on this observation, the patient repeated HRM and 24-hour esophageal impedance-pH monitoring 10 weeks after RFCA. HRM showed in all liquid swallows the typical hypercontractile contractions (with a greatest DCI $=15637$ $\mathrm{mmHg} \cdot \mathrm{cm} \cdot \mathrm{sec}$ ), consistent with the diagnosis of JE (Fig. 2), whereas impedance-pH monitoring resulted again negative for GERD. Patient started assumption of calcium-channel blockers (nifedipine drops $10 \mathrm{mg} /$ per os before meals), with a moderate dysphagia and chest-pain relieve. A mild weight gain was observed af-

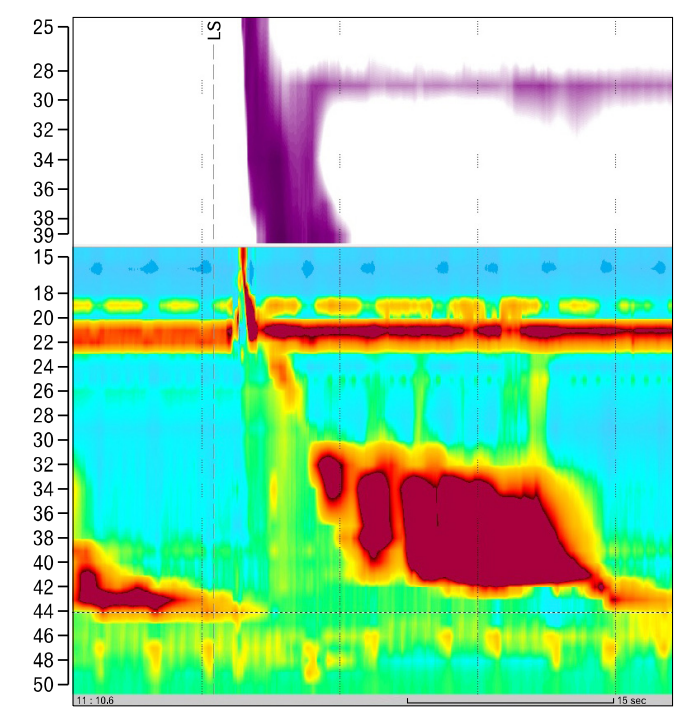

Figure 2. High-resolution manometry trace 8 weeks after radiofrequency catheter ablation for atrial fibrillation. In all liquid swallows the typical hypercontractile contractions were present (with a greatest distal contractile integral $=15637 \mathrm{mmHg} \cdot \mathrm{cm} \cdot \mathrm{sec}$ ), consistent with diagnosis of Jackhammer esophagus. ter 2 months ( $3 \mathrm{~kg}$ ). Given the persistency of symptoms and the weight loss patient is now under evaluation for endoscopic (botulin toxin injection and peroral endoscopic myotomy) or surgical approach (myotomy) for the treatment of dysmotility.

\section{Discussion}

To date, there is no available data regarding the impact of RFCA of left atrium on esophageal motility, whereas esophageal lesion, and in particular ulceration, due to thermal injury is a well-known complication of this procedure. ${ }^{6}$ In fact, development of esophageal ulcerations has been found to be dependent on the RFCA lesion set as well as the maximum energy delivered at the posterior left atrial wall. ${ }^{7}$ Also, some authors believe that esophageal ulceration can be a potential precursor of a dramatic life-threatening condition, the atrio-esophageal fistula. In order to reduce this complication, many attempts were made to protect the esophagus during RFCA by temperature monitoring, active cooling, or visualization of the esophageal course, but all have not proved to be effective. ${ }^{8}$

The potential damaging role of RFCA on the esophageal wall as well as on surrounding nerves (vagus and phrenic ones) was investigated in several papers. In a recent retrospective study, Knopp et $\mathrm{al}^{9}$ reviewed endoscopic findings of 425 patients who underwent RFCA for AF. All of them underwent upper endoscopy 1-3 days after radiofrequency procedure, and all of them were GERD-like or dysphagia-like symptom-free. Pathological findings were shown in $77 \%$ of the patients, including esophagitis and chronic gastritis. However, thermal lesions were found in $11 \%$ of cases and gastroparesis in $17 \%$, supporting the potential role of RFCA in damaging esophagus and the near vagus nerve. Furthermore, a previous study has reported that within 48 hours after AF ablation abdominal pain and distension developed, even if in only $1 \%$ of patients, ${ }^{10}$ and a case report of severe gastroparesis was published recently. ${ }^{11}$ The pathophysiology of JE remains unclear but different hypotheses have been suggested. Hypertensive contractions may be related to muscular hypertrophy secondary to mechanical outflow obstruction, as JE have been descripted in patients with dysphagia after fundoplication or gastric band. ${ }^{12}$ However, in our case, patient showed a normal esophageal anatomy, without stricture or difficult in transit through esophagogastric junction. Another possible hypothesis takes in account GERD, that can be associated with chest pain and hypertensive esophageal contractions. The prevalence of GERD is observed to be higher after RFCA. Martinek et $\mathrm{al}^{2}$ 
evaluated patients before and after RFCA with upper endoscopy and $\mathrm{pH}$ monitoring. They reported the incidence of "de novo" GERD in $19 \%$ of patients after procedure, arguing that reflux rising could be attributable to a direct vagal stimulation, that can lead to a decrease in lower esophageal sphincter tone. Interestingly, Khan et $\mathrm{al}^{13}$ reported a case of JE triggered after lung transplantation. The young female had an objective GERD documented at impedance-pH monitoring, and JE responded to PPI. Also, patient reported to have significant gastroparesis; authors concluded that after lung transplantation, vagus nerves can be injured near the lung hila, and this disturbance could lead to esophageal dysmotility and delayed gastric emptying.

Further, we supposed that JE in our patient was triggered by a direct stimulus to vagal nerve. This latter, in fact, is adjacent to the heart, and a thermic damage with RFCA is possible, as vagal injure causing delayed gastric emptying is considered a direct noncardiac complication of the ablation procedure. However, even if a gastric emptying test was not performed, our patient did not show any sign of delayed gastric emptying. Upper endoscopy did not found food retention in stomach, neither patient reported abdominal pain, dumping, nausea, vomiting, or any kind of dyspeptic-like symptom. Actually, gastroparesis is supposedly caused by damage to the vagal nerve fibers surrounding the distal esophagus, as suggested in previous studies. ${ }^{12}$ Furthermore, Sifrim et $\mathrm{al}^{14}$ have suggested that the mechanism of the increase in esophageal contraction amplitude might be due to an imbalance between excitatory and inhibitory innervation, and that patients with spastic disorders of the esophagus have an impaired inhibitory innervation, whether this abnormality can be secondary to the vagal nerves denervation. Thus, we hypothesized that in our patient RFCA induced a thermal lesion to vagal nerve fibers only surrounding mid esophagus. This event created an imbalance in favor of excitatory innervation, causing the high-amplitude repetitive contractions distinctive of $\mathrm{JE}$.

In summary, the pre-RFCA evaluation of this patient did not show any esophageal abnormality, whereas the JE manifested after the radiofrequency ablation, and partially recovered with calcium channel blockers. The pathophysiology of this rare esophageal dysmotility is still unclear, and different possible etiopathogenesis can be represented by GERD, by outflow obstruction in and around LES, by drugs or by a direct injury to vagal nerve. The latter was the most likely mechanism eliciting JE, supporting the hypothesis of direct thermal damage. Esophageal dysmotility can represent a possible complication of RFCA for $\mathrm{AF}$, and dysphagia appearance after this procedure must be timely investigated by HRM.

\section{References}

1. Pappone C, Oral H, Santinelli V, et al. Atrio-esophageal fistula as a complication of percutaneous transcatheter ablation of atrial fibrillation. Circulation 2004;109:2724-2726.

2. Martinek M, Hassanein S, Bencsik G, et al. Acute development of gastroesophageal reflux after radiofrequency catheter ablation of atrial fibrillation. Heart Rhythm 2009;6:1457-1462.

3. Roman S, Pandolfino JE, Chen J, Boris L, Luger D, Kahrilas PJ. Phenotypes and clinical context of hypercontractility in high-resolution esophageal pressure topography (EPT). Am J Gastroenterol 2012;107:37-45.

4. Pandolfino JE, Ghosh SK, Rice J, Clarke JO, Kwiatek MA, Kahrilas PJ. Classifying esophageal motility by pressure topography characteristics: a study of 400 patients and 75 controls. Am J Gastroenterol 2008;103:27-37.

5. Kahrilas PJ, Bredenoord AJ, Fox M, et al. International high resolution manometry working group. The Chicago Classification of esophageal motility disorders, v3.0. Neurogastroenterol Motil 2015; 27:160-174.

6. Marrouche NF, Guenther J, Segerson NM, et al. Randomized comparison between open irrigation technology and intracardiac-echoguided energy delivery for pulmonary vein antrum isolation: procedural parameters, outcomes, and the effect on esophageal injury. J Cardiovasc Electrophysiol 2007;18:583-588.

7. Martinek M, Bencsik G, Aichinger J, et al. Esophageal damage during radiofrequency ablation of atrial fibrillation: impact of energy settings, lesion sets, and esophageal visualization. J Cardiovasc Electrophysiol 2009;20:726-733.

8. Martinek M, Purerfellner H. Nightmares in atrial fibrillation ablation-identification, management, and prevention of complications in radiofrequency ablation of atrial fibrillation. Herzschrittmacherther Elektrophysiol 2007;18:216-224.

9. Knopp H, Halm U, Lamberts R, et al, Incidental and ablation-induced findings during upper gastrointestinal endoscopy in patients after ablation of atrial fibrillation: a retrospective study of 425 patients. Heart Rhythm 2014;11:574-578.

10. Shah D, Dumonceau J, Burri H, et al. Acute pyloric spasm and gastric hypomotility: an extracardiac adverse effect of percutaneous radiofrequency ablation for atrial fibrillation. J am Coll Cardiol 2005; 46:327-330.

11. Choi SW, Kang SH, Kwon OS, et al. A case of severe gastroparesis: indigestion and weight loss after catheter ablation of atrial fibrillation. Pacing Clin Electrophysiol 2012;35:e59-e61.

12. Burton PR, Brown W, Laurie C, et al. The effect of laparoscopic adjustable gastric bands on esophageal motility and the gastroesophageal junction: analysis using high-resolution video manometry. Obes Surg 2009;19:905-914.

13. Khan MQ, Nizami IY, Khan BJ, Al-Ashgar HI. Lung transplantation triggered "jackhammer esophagus": a case report and review of literature. J Neurogastroenterol Motil 2013;19:390-394.

14. Sifrim D, Janssens J, Vantrappen G. Failing deglutitive inhibition in primary esophageal motility disorders. Gastroenterology 1994;106: 875-882. 